\title{
A INFLUÊNCIA DAS MALFORMAÇÕES CARDÍACAS CONGÊNITAS DE CRIANÇAS COM SÍNDROME DO CROMOSSOMO 21
}

Caroline Nunes Gonzaga ${ }^{1}$, Larissa Borba André2, Mileide Cristina Stoco de Oliveira², Silas de Oliveira Damasceno $^{3}$, Katiane Mayara Guerrero ${ }^{3}$, Carla de Oliveira Carletti ${ }^{2}$, Alline Sayuri Tacaki Alves ${ }^{1}$, Augusto Cesinando de Carvalho ${ }^{4}$, Tânia Cristina Bofi ${ }^{4}$.

Universidade Estadual Paulista - UNESP, ${ }^{1}$ Curso de Fisioterapia, ${ }^{2}$ Pós-Graduação em Fisioterapia, Residência em Saúde, ${ }^{3}$ Pós-Graduação em Fisioterapia, Especialização em Neurologia, ${ }^{4}$ Departamento de Fisioterapia, Presidente Prudente, SP. e-mail: caa nunes 11@yahoo.com.br

\section{RESUMO}

As malformações cardíacas afetam entre 40 e $50 \%$ dos casos com Síndrome de Down (SD) enquanto sua prevalência no recém-nascido sem SD varia de 40 a 60\%. Existe uma incidência de cardiopatias congênitas (CC) significativamente superior nas mulheres com SD. Sendo assim, o objetivo do presente estudo foi comparar o desenvolvimento motor de bebês com SD de zero a quatro meses de idade que apresentavam ou não CC. Participaram 26 crianças com SD, com idade média de 3,84 $\pm 3,28$ meses, frequentadoras de um centro de atendimento de fisioterapia e reabilitação da UNESP. Foi utilizado como instrumento o Inventário Portage Operacionalizado (IPO) e a anamnese realizada com os pais e posteriormente uma análise descritiva dos dados utilizando média, desvio padrão e porcentagem. Concluiu-se que o fator de risco cardiopatia não foi determinante para o atraso no desenvolvimento neuropsicomotor.

Palavras-chave: Síndrome de Down, cardiopatias congênitas, desenvolvimento infantil, habilidades motoras, desvios do desenvolvimento infantil.

\section{THE INFLUENCE OF CONGENITAL CARDIAC CONGENITAS OF CHILDREN WITH SYNDROME OF CHROMOSOME 21}

\begin{abstract}
The cardiac malformations affect between 40 and $50 \%$ of the cases with Down syndrome (DS) as its prevalence in the newborn without DS varies from 40 to $60 \%$. There is an incidence of congenital heart defects (CC) was significantly higher in women with DS. Thus, the objective of this study was to compare the motor development of infants with DS from zero to four months of age who presented or not CC. Participated in 26 children with DS, with an average age of $3.84 \pm 3.28$ months, attendant a center for care on physical therapy and rehabilitation of UNESP. It was used as an instrument the Inventory Portage Operationalized (IPO) and the interview held with parents and subsequently a descriptive data analysis using mean, standard deviation and percentage. It was concluded that the risk factor for heart disease was not a determinant for the delay in psychomotor development.
\end{abstract}

Keywords: Down Syndrome, heart defects, child development, motor skills, deviations of child development.

\section{INTRODUÇÃO}

A Síndrome de Down (SD) é a mais comum condição genética não hereditária, caracterizada pela alteração do cromossomo 21 que ocorre no início da gravidez, sendo que os tipos mais encontrados na literatura são: translocação, mosaicismo e trissomia simples, este último com maior incidência ${ }^{1,2}$.

O Censo 2010 do IBGE (Instituto Brasileiro de Geografia e Estatística) estima que 300 mil tenham SD, que ocorre com uma prevalência de 1 para cada 600 nascimentos aproximadamente ${ }^{3}$.

O principal fator de risco para a ocorrência da síndrome é a idade materna avançada, notando-se aumento significativo a partir dos 35 anos, e com incidência de 1 para cada 30 nascidos vivos de mães com mais de 45 anos $^{2}$. Alguns estudos mostram que a idade paterna avançada também pode ser um fator contribuinte 4 . Não existe evidência da ação de fatores hormonais, deficiências de vitaminas, 
drogas, toxinas, vírus ou fatores racial, social e econômico sobre a incidência da síndrome, nem dados de predileção por sexo².

As manifestações fenotípicas mais comuns são a deficiência intelectual, hipotonia muscular, baixa estatura, anomalias faciais, mãos curtas, frequentemente com prega palmar única e malformações congênitas, especialmente as cardíacas ${ }^{4}$.

Embora muitas das malformações citadas possam definir o quadro clínico dessas crianças, as cardiopatias congênitas (CC) são as condições que influenciam diretamente tanto o prognóstico quanto a sobrevida, sendo a maior causa de morbidade e mortalidade nos primeiros dois anos de vida ${ }^{2,5}$.

As malformações cardíacas afetam entre 40 e $50 \%$ dos casos com SD enquanto sua prevalência no recém-nascido sem SD varia de 40 a $60 \%$. Existe uma incidência de CC significativamente superior nas mulheres com SD comparadas aos homens. Dentre as CC destacamse o prolapso mitral, defeitos do septo átrioventricular, ventricular e atrial, persistência do ducto arterial e tetralogia de Fallot ${ }^{6}$. Quanto mais tardiamente ocorrer a indicação cirúrgica da CC, pior será o prognóstico da criança².

Portanto, devido à alta incidência de comprometimento cardíaco, preconiza-se que todas as crianças, mesmo as assintomáticas, sejam submetidas ao ecocardiograma durante os primeiros seis meses de vida, considerando que as CC são as causas mais comuns de morte até um ano de idade ${ }^{6}$.

Além dos comprometimentos mencionados, a criança com SD apresenta também atraso neuropsicomotor. Algumas habilidades motoras como controle cefálico, rolar, sentar, arrastar engatinhar e deambular, são adquiridas mais tardiamente quando comparadas as crianças com desenvolvimento típico ${ }^{7}$. Enquanto bebês típicos, em média, sentam independentemente, ficam em pé sozinhos e andam com 6, 11 e 12 meses de idade, respectivamente, bebês com síndrome de Down fazem o mesmo com 9, 18 e 19 meses. Sendo que a média de alcance do andar independente na SD pode variar de 22,3 a 30 meses, em comparação com as crianças não sindrômicas, em que a média é de 11,7 meses $^{6}$. Estas limitações motoras são observadas predominantemente na primeira infância e podem influenciar a capacidade da criança desempenhar independentemente as atividades de vida diária ${ }^{7}$.
Muitas escalas buscam avaliar crianças com SD a fim de verificar seu desenvolvimento neuropsicomotor. $\mathrm{O}$ Inventário Portage Operacionalizado (IPO) ${ }^{8}$ é um dos instrumentos utilizados na literatura que trouxe grande contribuição, pois permite avaliar crianças de zero a 6 anos em seis domínios (estimulação infantil, socialização, linguagem, autocuidados, cognição e desenvolvimento motor), com o intuito de realizar uma intervenção precoce baseado em seus resultados ${ }^{9,10}$.

A intervenção precoce em crianças com SD é essencial, pois atua em acelerar o ganho das habilidades motoras grossas, proporcionando maior interação e exploração do ambiente com mais funcionalidade e independência. Um dos objetivos da fisioterapia no tratamento dessas crianças é aumentar a ativação muscular promovendo o controle postural antigravitacional ${ }^{11}$.

Dessa forma, devido aos comprometimentos que a SD pode gerar, em especial o surgimento de CC, evidencia-se a importância de se verificar o quanto as CC interferem no desenvolvimento neuropsicomotor de crianças com SD. O impacto das CC no desenvolvimento neuropsicomotor de pacientes com essa síndrome tem sido pouco elucidado na literatura, fazendo-se necessário surgir mais pesquisas com essa abordagem, visto que um melhor entendimento dessas alterações cardíacas podem interferir nas condutas terapêuticas ${ }^{6,11}$.

Sendo assim, o objetivo do presente estudo foi comparar o desenvolvimento neuropsicomotor de bebês com síndrome de Down de zero a quatro meses de idade que apresentavam ou não cardiopatias congênitas.

\section{MÉTODO}

Trata-se de uma pesquisa descritiva, aprovada pelo comitê de ética e pesquisa sobre o CAEE: 25887913.0.0000.5402 da Faculdade de Ciências e Tecnologia (FCT) da UNESP, campus de Presidente Prudente. Para participar da presente pesquisa os seguintes critérios de elegibilidade foram respeitados: diagnóstico clínico de síndrome de Down por trissomia do cromossomo 21, estar em atendimento em um centro de atendimento de fisioterapia e reabilitação da FCT - UNESP. A amostragem composta por 26 crianças com SD de ambos os sexos, foram analisados prontuários dos pacientes atendidos no centro e a anamnese realizada com os pais no 
momento em que as crianças entraram no setor de neurologia do centro, averiguando quais apresentavam cardiopatias congênitas e quais eram as cardiopatias.

O instrumento utilizado para avaliar o desenvolvimento neuropsicomotor foi $O$ Inventário Portage Operacionalizado (IPO), descrito por Williams e Aiello ${ }^{8}$, que fornece uma visão global do desenvolvimento da criança (de zero a seis anos de idade) dividindo-o em seis domínios, tais como estimulação infantil, socialização, linguagem, autocuidados, cognição e desenvolvimento motor, possibilitando a detecção de áreas que devem ser objeto de intervenção. Dentre essas, foi usada a estimulação infantil, que consiste em avaliar os movimentos e habilidades do bebê de zero a quatro meses. Esse protocolo avalia 45 comportamentos divididos em estimulação ambiental, observação e comportamento ${ }^{12}$.

O IPO utiliza como critério de desempenho significativo para cada área a ocorrência de um acerto de no mínimo $75 \%$ em determinada faixa etária ${ }^{12}$. Foi realizada posteriormente uma análise descritiva dos dados utilizando média, desvio padrão e porcentagem.

\section{RESULTADOS}

Foram avaliadas 26 crianças com SD, sendo 17 meninas e 9 meninos, com idade média de $3,84 \pm 3,28$ meses.

Dessas, $\quad 57,69 \% \quad(15)$ apresentavam cardiopatias (Figura 1), sendo que $47 \%$ tinham o defeito na comunicação interatrial (CIA), 6\% comunicação interventricular (CIV), 20\% CIA e CIV associados e $27 \%$ outras cardiopatias encontradas tais como: cavidade direita discretamente dilatada, displasia conjunta da válvula tricúspide, persistência do canal ventricular, defeito no septo átrio ventricular total tipo A.

Todas as crianças apresentaram atraso no desenvolvimento neuropsicomotor correspondente a sua idade cronológica, das que apresentavam cardiopatia, 53,33\% apresentaram atrasos significativos (abaixo do esperado para idade) e das que não apresentavam cardiopatias, $75 \%$ apresentaram atrasos significativos.

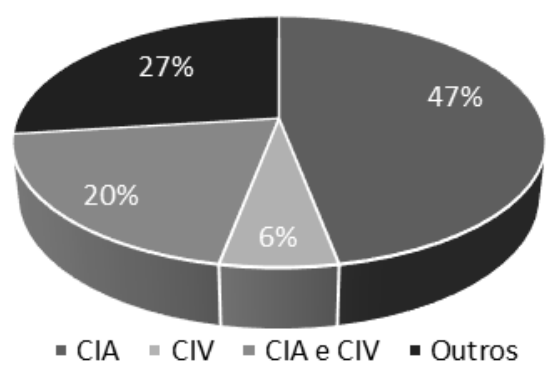

Figura 1. Proporções de cardiopatias observadas nas crianças. $\mathrm{CIA}$ - defeito na comunicação interatrial, CIV - defeito na comunicação interventricular.

\section{DISCUSSÃO}

Crianças com síndrome de Down apresentam uma lentificação na aquisição das habilidades motoras das reações posturais de proteção, retificação e equilíbrio, assim como também apresentam comprometimentos na cognição, comunicação e desenvolvimento psicossocial $^{6}$.

Este estudo evidenciou que crianças com síndrome de Down apresentam comprometimento no desenvolvimento neuropsicomotor, porém nem todas possuem cardiopatias. Um estudo que avaliou 24 crianças com SD divididas em grupo cardiopatas e não cardiopatas encontrou como resultado atraso no desenvolvimento em $60 \%$ dos avaliados do primeiro grupo e em $88 \%$ no segundo ${ }^{13}$, que por sua vez corrobora com os resultados obtidos no presente estudo, pois não cardiopatas apresentaram maior atraso no desenvolvimento quando comparados aos cardiopatas, considerando assim que a cardiopatia não foi um fator determinante para $o$ atraso no desenvolvimento neuropsicomotor.

Os avanços tecnológicos têm propiciado correções cirúrgicas que aumentam a sobrevida das crianças com cardiopatia e melhora da expectativa de vida deste grupo populacional, mas a prevalência de alterações cardíacas nessa população paira em torno de $40 \%$ a $50 \%{ }^{4}$. No atual estudo, esse percentual encontrou-se dentro da estimativa preconizada na literatura.

Em relação ao tipo mais recorrente de cardiopatia, um estudo realizado para analisar as caraterísticas e prevalência de cardiopatias congênitas em crianças com SD submetidas à cirurgia cardíaca encontrou os seguintes dados: Defeito do Septo Atrioventricular (DSAV - 27,8\%), comunicação interventricular (CIV - 14,9\%) e 
comunicação interatrial (CIA - 10,6\%) dos $\operatorname{casos}^{14}$. Os dados diferem dos encontrados no presente estudo, na qual a maior prevalência foi de CIA em $47 \%$.

No vigente estudo houve um aglutino de cardiopatias, o que confirma com outro estudo que também mostra que a comunicação interatrial e a comunicação interventricular são os tipos de CC mais frequentes, entretanto neste estudo o percentual ficou em $31 \%^{2}$, dado acima do apresentado no atual estudo.

Esta pesquisa apresenta limitações, uma vez que não se sabe se todos os pais aderiram às orientações terapêuticas, ou seja, algumas crianças podem ter sido estimuladas mais do que as outras, o que poderia alterar os resultados, assim como o número da amostra é pequeno, entretanto o tema se torna relevante devido à elevada prevalência dessas cardiopatias nesta população no estudo em questão e por ser pouco elucidado na literatura.

\section{CONCLUSÃO}

Os resultados obtidos no presente estudo apontam que o fator de risco da CC não foi determinante para o atraso no desenvolvimento neuropsicomotor. Entretanto os resultados negativos no desenvolvimento dependem não somente de um, mas de uma combinação de vários fatores de risco. Sendo assim, é de suma importância a averiguação principalmente da quantidade do que da natureza desses fatores de risco já que estes são incididos diretamente no desenvolvimento.

\section{CONFLITO DE INTERESSE}

Os autores declaram não haver qualquer potencial de conflito de interesse que possa interferir na imparcialidade deste trabalho científico.

\section{REFERÊNCIAS}

1. Godzicki B, Silva PA, Blume LB. Aquisição do sentar independente na Síndrome de Down utilizando o balanço. Fisioter Mov. 2010;23(1):7381. DOI: http://dx.doi.org/10.1590/S010351502010000100007

2. Júnior HLB, Guedes SS, Noronha FL, Júnior TJS. Prevalência de cardiopatia congênita em crianças com síndrome de Down de Juiz de Fora e região. HU Revista. 2011;37(2):147-53.
3. IBGE - Instituto Brasileiro de Geografia e Estatística. Censo Demográfico 2010. Disponível em: http://www.ibge.gov.br

4. Valenzuela NJB, Passarelli MLB, Coates MV, Nascimento LFC. Recuperação pôndero-estatural em crianças com síndrome de Down e cardiopatia congênita. Rev Bras Cir Cardiovasc. 2011;26(1):61-8.

DOI:

http://dx.doi.org/10.1590/S010276382011000100013

5. Mourato FA, Villachan LRR, Mattos SS. Prevalência e perfil das cardiopatias congênitas e hipertensão pulmonar na síndrome de Down em serviço de cardiologia pediátrica. Rev Paul Pediatr. 2014;32(2):159-63. DOI: http://dx.doi.org/10.1590/01030582201432218913

6. HoepersI A, Schenkel IC, Schivinski CIS. Cardiopatia e desenvolvimento motor na Síndrome de Down: série de casos. Arq Catarin Med. 2013;42(2):86-92.

7. Agostini B, Bisognin JP, Martins JS. Avaliação funcional de crianças com síndrome de Down por meio do Inventário de avaliação pediátrica de incapacidade. Ciên Saúde. 2013;14(2):209-16.

8. Williams LCA, Aiello ALR. O Inventário Portage operacionalizado: intervenção com famílias. São Paulo: Memnon; 2001

9. Formiga CK, Pedrazzani ES, Silva FPS, Lima CD. Eficácia de um programa de intervenção precoce com bebês pré-termo. Paidéia. 2004;14(29):30111. DOI: http://dx.doi.org/10.1590/S0103863X2004000300006

10. Anunciação LMRL, Costa MPR, Denari FE. Educação infantil e práticas pedagógicas para o aluno com Síndrome de Down: o enfoque no desenvolvimento motor. Rev Bras Ed Esp. 2015;21(2):229-44. DOI: http://dx.doi.org/10.1590/S141365382115000200005

11. Garcia C, Alvarez CDL. Efeito do treino de marcha em esteira em crianças com Síndrome de Down com e sem cardiopatia. Saúde (Batatais). 2014;3(1):59-72.

12. Barcellos, EM, Bertini MT, Lima TS et al. A utilização do Inventário Portage como instrumento de avaliação no serviço de aconselhamento genético. VIII encontro da associação brasileira de pesquisadores em educação especial $2013 . \quad$ Dispoinvel: http://www.uel.br/eventos/congressomultidiscip linar/pages/arquivos/anais/2013/AT132013/AT13-011.pdf 
13. André LB, Fajalle FM, Oliveira MCS, Mata LM, Bofi TC. Crianças com síndrome de Down com cardiopatia congênita apresentam maior atraso no desenvolvimento neuropsicomotor? Trabalho apresentado no VII Congresso Brasileiro Sobre Síndrome de Down, Curitiba, 2015. Disponível em:

http://down2015.com.br/site/wpcontent/uploads/2014/11/Anais-

CongressoCientifico.pdf

14. Doná TCK, Lawina B, Maturana CS, Felcara JM. Características e prevalência de cardiopatias congênitas em crianças com Síndrome de Down submetidas à cirurgia cardíaca em um hospital na região norte do Paraná. Rev Equil Corp Saúde. 2015;7(1):11-6.

Recebido para publicação em 17/08/2016

Revisado em 21/08/2016

Aceito em 23/08/2016 